\title{
Higher-order condensate corrections to bottomonium observables
}

\section{Thomas Rauh*}

Institute for Particle Physics Phenomenology, Department of Physics, University of Durham, DH1 3LE, United Kingdom,

Albert Einstein Center for Fundamental Physics, Institute for Theoretical Physics, University of Bern, Sidlerstrasse 5, CH-3012 Bern, Switzerland

E-mail: rauheitp.unibe.ch

The perturbative accuracy for bottomonium observables has recently been extended to next-tonext-to-next-to-leading order. Assuming the hierarchy $\Lambda_{\mathrm{QCD}} \ll m_{b} v^{2}$ holds, non-perturbative effects take the form of local condensates. I determine higher-order corrections in this approach and assess its validity by studying the convergence of the series. In particular, the non-perturbative effects on the determination of the bottom-quark mass from the bottomonium spectrum and sum rules are discussed.

XIII Quark Confinement and the Hadron Spectrum - Confinement2018

31 July - 6 August 2018

Maynooth University, Ireland

${ }^{*}$ Speaker. 


\section{Introduction}

Bottomonium provides a rich environment for studying QCD in a non-relativistic setting where the three-velocity $v$ of the bottom quarks is of the same order as the strong coupling constant $\alpha_{s} \ll 1$. This implies a hierarchy between the energy $m_{b} v^{2}$, the momentum $m_{b} v$ and the mass $m_{b}$ of the bottom quarks and bottomonium observables receive contributions from several momentum modes [1]

$$
\begin{array}{lll}
\text { hard: } & k^{0} \sim m_{b}, & \mathbf{k} \sim m_{b}, \\
\text { soft: } & k^{0} \sim m_{b} v, & \mathbf{k} \sim m_{b} v, \\
\text { potential: } & k^{0} \sim m_{b} v^{2}, & \mathbf{k} \sim m_{b} v, \\
\text { ultrasoft: } & k^{0} \sim m_{b} v^{2}, & \mathbf{k} \sim m_{b} v^{2} .
\end{array}
$$

This challenging multiscale problem is further complicated by the presence of so-called Coulomb singularities $\left(\alpha_{s} / v\right)^{n} \sim 1$ which are caused by ladder-type exchanges of potential (or Coulomb) gluons shown in Figure 1. These effects are non-perturbatively strong in the non-relativistic count-

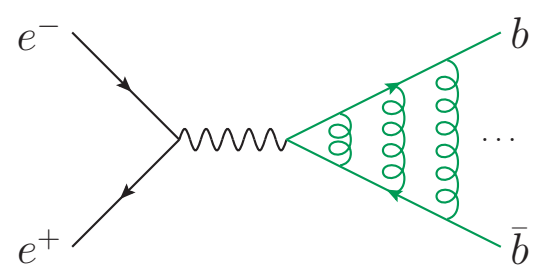

Figure 1: Ladder exchanges of potential gluons in the process $e^{+} e^{-} \rightarrow b \bar{b}$ near the $b \bar{b}$ production threshold $s \sim 4 m_{b}^{2}$ where the bottom quark momenta are in the potential region, i.e. non-relativistic.

ing $v \sim \alpha_{s} \ll 1$ and, when resummed to all orders, produce bound-state poles in the $e^{+} e^{-} \rightarrow b \bar{b}$ cross section that correspond to the $\Upsilon(N S)$ states. The effective theory potential non-relativistic $Q C D$ (PNRQCD) [2-5] provides a framework to perform the resummation of the Coulomb singularities and to systematically determine higher-order contributions, and its development has paved the way for perturbative calculations at the next-to-next-to-next-to-leading order (NNNLO) in the non-relativistic counting $v \sim \alpha_{s}$. Results at this order have recently been obtained for the spectrum [6], the leptonic decay $\Upsilon(1 S) \rightarrow l^{+} l^{-}$[7] and the large- $n$ moments of the $e^{+} e^{-} \rightarrow b \bar{b} X$ cross section [8].

PNRQCD is constructed by subsequently integrating out the hard scale $m_{b}$ and the soft scale $m_{b} v$ and only contains potential bottom quarks $\psi$ and ultrasoft massless modes (gluons, light quarks and ghosts) as dynamical degrees of freedom. This splits the computation into hard matching calculations (see e.g. [9]), soft matching calculations (see e.g. [10]) and the calculation of a nonrelativistic Green function $G(E)$ in the effective theory $[5,11,12]$. The effective Lagrangian for the latter takes the form

$$
\begin{aligned}
\mathscr{L}_{\mathrm{PNRQCD}}= & \psi^{\dagger}\left(i \partial_{0}+\frac{\partial^{2}}{2 m}+g_{s} A_{0}(t, \mathbf{0})-g_{s} \mathbf{x} \cdot \mathbf{E}(t, \mathbf{0})+\frac{\partial^{4}}{8 m^{3}}\right) \psi+\mathscr{L}_{\text {antibottom }} \\
& +\int d^{d-1} \mathbf{r}\left[\psi_{a}^{\dagger} \psi_{b}\right](x+\mathbf{r}) V_{a b ; c d}(\mathbf{r}, \partial)\left[\chi_{c}^{\dagger} \chi_{d}\right](x)+\mathscr{L}_{\text {ultrasoft }},
\end{aligned}
$$


where the interaction between the potential bottom-quarks and the ultrasoft gluons has been multipole expanded in the first line and the second line contains the spatially non-local interactions between the bottom-antibottom pair which, at leading order, are given by the color-Coulomb potential which corresponds to the ladder exchanges of potential gluons. The last term is a copy of the QCD Lagrangian which only contains the ultrasoft modes.

Perturbative calculations at NNNLO all implicitly assume that the QCD scale $\Lambda_{\mathrm{QCD}}$ is much smaller than the ultrasoft scale $m_{b} v^{2}$. In this situation, first studied by Voloshin and Leutwyler [1821], non-perturbative effects appear as a series of local vacuum condensates which are suppressed by increasing powers of $\Lambda_{\mathrm{QCD}} /\left(m_{b} v^{2}\right)$. While the hierarchy is clearly justified for the production of top quark pairs near threshold [13-17], we can at best hope that it is valid for a subset of observables in the bottomonium system. In these proceedings, I present recent work [22] where the convergence of this approach is assessed by considering higher-order corrections in the expansion in $\Lambda_{\mathrm{QCD}} /\left(m_{b} v^{2}\right)$. The phenomenological discussion centers around the determination of the bottom-quark mass from the bottomonium spectrum or sum rules.

\section{Non-perturbative effects as local condensates}

When $\Lambda_{\mathrm{QCD}} \ll m_{b} v^{2}$, the gluon field in the Lagrangian (1.1) can be split $A_{\mu}(t, \mathbf{x})=A_{\mu}^{\text {us }}(t, \mathbf{x})+$ $A_{\mu}^{\mathrm{np}}(t, \mathbf{x})$. The leading charge term in the multipole expansion does not contribute which can be seen immediately by choosing Fock-Schwinger gauge where $\mathbf{x} \cdot \mathbf{A}^{\mathrm{np}}(t, \mathbf{x})=0$ and $A_{0}^{\mathrm{np}}(t, \mathbf{0})=0$. The first non-vanishing interaction is the chromoelectric dipole term

$$
\mathscr{L}_{\text {non-perturbative }}=\psi^{\dagger}\left(-g_{s} \mathbf{x} \cdot \mathbf{E}^{\mathrm{np}}(0, \mathbf{0})+\ldots\right) \psi+[\text { antibottom }]
$$

which can be treated as a perturbation. The non-relativistic Green function

$$
G(E) \equiv\left\langle\mathbf{0}\left|(\hat{H}-E-i 0)^{-1}\right| \mathbf{0}\right\rangle,
$$

must then be computed with the Hamiltonian

$$
\hat{H}=\hat{H}_{b \bar{b}}+\hat{H}_{\mathrm{np}}+\hat{H}_{D}+\ldots
$$

where $\hat{H}_{b \bar{b}}$ is the perturbative bottomonium Hamiltonian, $\hat{H}_{\mathrm{np}}$ is the Hamiltonian for the nonperturbative degrees of freedom and $\hat{H}_{D}$ corresponds to the dipole interaction (2.2). Since the latter is a perturbation the state $|\mathbf{0}\rangle$ factorizes into a bottom-antibottom state $|\mathbf{0}\rangle_{b \bar{b}}$ at zero spatial distance and the non-perturbative QCD vacuum state $|0\rangle_{\mathrm{np}}$. Expansion in $\Lambda_{\mathrm{QCD}}$ yields

$$
G(E)=\left\langle\mathbf{0}\left|\hat{G}_{b \bar{b}}^{(1)}(E)\right| \mathbf{0}\right\rangle_{b \bar{b}}+\sum_{n=0}^{\infty}\left\langle\mathbf{0}\left|\hat{G}_{b \bar{b}}^{(1)}(E) \hat{x}^{i}\left[\hat{G}_{b \bar{b}}^{(8)}(E)\right]^{1+2 n} \hat{x}^{i} \hat{G}_{b \bar{b}}^{(1)}(E)\right| \mathbf{0}\right\rangle_{b \bar{b}} O_{n}+\ldots,
$$

where

$$
\hat{G}_{b \bar{b}}(E)=\left(\hat{H}_{b \bar{b}}-E-i 0\right)^{-1}
$$

is the perturbative Green function and the vacuum condensates take the form

$$
O_{n}=\left\langle 0\left|\frac{g_{s}^{2}}{18}\left(E^{\mathrm{np}}\right)_{i}^{A}\left[\hat{H}_{\mathrm{np}}\right]^{2 n}\left(E^{\mathrm{np}}\right)_{i}^{A}\right| 0\right\rangle_{\mathrm{np}}
$$



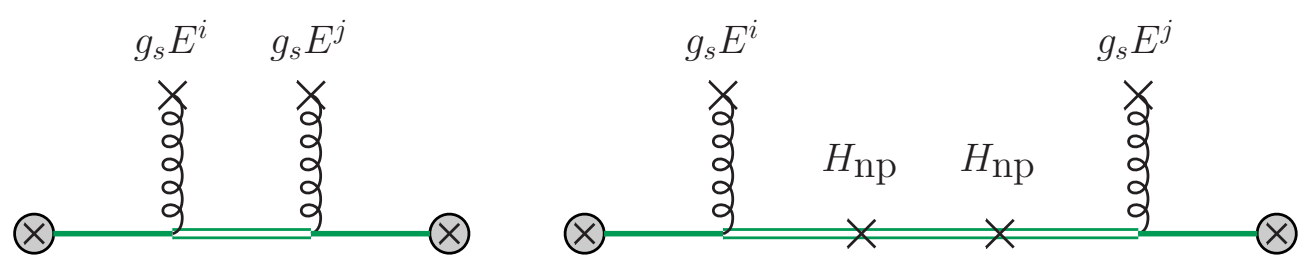

Figure 2: Feynman graphs corresponding to the first two terms in the sum (2.4). Single and double lines denote the color-singlet and color-octet Green functions. Higher orders in the expansion follow by inserting additional pairs of $\hat{H}_{\text {np }}$ between the two insertions of the dipole $\hat{H}_{D}$.

The Feynman graph representation for the non-perturbative contributions is shown in Figure 2. The first term in the sum in (2.4) has dimension four and the corresponding condensate $O_{0}$ reduces to the gluon condensate $\left\langle\frac{\alpha_{s}}{\pi} G^{2}\right\rangle$. This term has already been studied 40 years ago [18-21]. The next term is of dimension six and has first been computed in [23]. In [22], I have determined the correction of dimension eight and partial NLO QCD corrections from the insertion of the NLO Coulomb potential to the dimension four terms, c.f. Figure 3. The determination of ultrasoft corrections needed to achieve full NLO accuracy is postponed to future work. Details about the calculations and analytical results have been presented in [22].
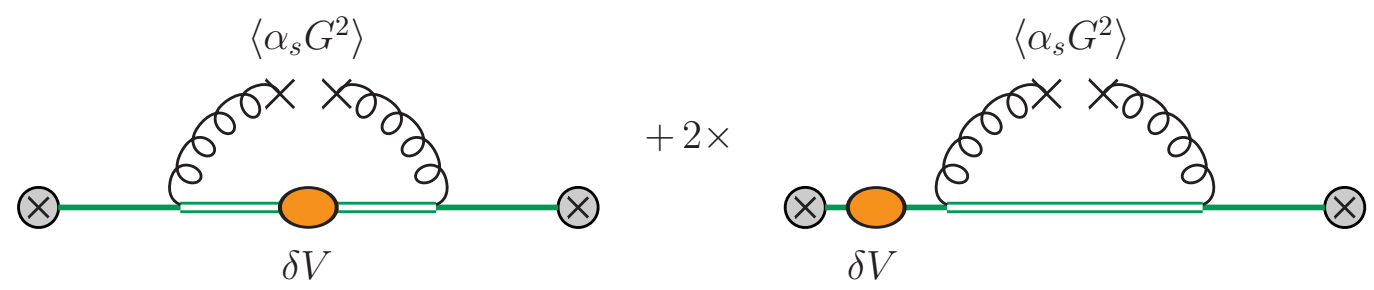

Figure 3: Corrections to the dimension four condensate contribution from an insertion of the NLO Coulomb potential $\delta V$. Ultrasoft effects from the diagrams shown in [22] contribute at the same order.

\section{Phenomenology}

The size of non-perturbative contributions in bottomonium has been strongly disputed as illustrated in Figure 4 where an overview over determinations of the bottom-quark mass from the spectrum is presented together with the various error estimates for the non-perturbative contribution. The recent tendency to neglect this source of uncertainty altogether was part of the motivation for this work. The reason why there has never been consensus about the non-perturbative contribution is that even the size of the leading-order dimension four part is far from certain. First of all, the value of the gluon condensate is poorly known [24]. In this analysis we use the SVZ value [25] for the gluon condensate which translates to $O_{0}^{\mathrm{SVZ}}=-(285 \mathrm{MeV})^{4}$ and apply a naive rescaling for the higher dimensions: $O_{1}^{\text {naive }}=(285 \mathrm{MeV})^{6}$ and $O_{2}^{\text {naive }}=-(285 \mathrm{MeV})^{8}$. To account for uncertainties we multiply these values with factors that are varied between 0 and 3,0 and $3^{3 / 2}$, and 0 and $3^{2}$, respectively. Secondly, the condensate corrections are strongly scale dependent. For instance the 


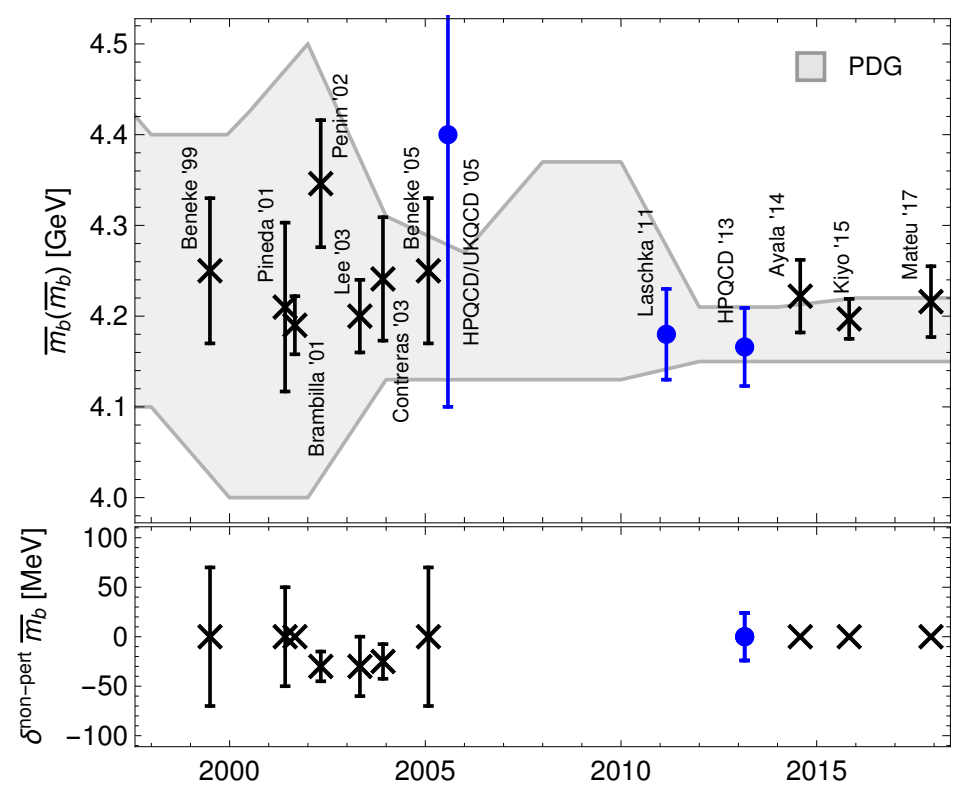

Figure 4: Historical overview of determinations of the bottom-quark $\overline{\mathrm{MS}}$ mass from the bottomonium spectrum. The bottom panel shows the non-perturbative correction including the uncertainty estimate where one was provided. The blue points are based on lattice simulations.

dimension-four contribution to the $\Upsilon(N S)$ mass takes the form

$$
\delta_{\Lambda_{\mathrm{QCD}}^{4}} M_{\Upsilon(N S)}=-\frac{m_{b} \alpha_{s}^{2}\left(\mu_{c}\right) C_{F}^{2}}{4 N^{2}}\left(\tilde{e}_{N}^{(4,0)}+\frac{\alpha_{s}\left(\mu_{c}\right)}{4 \pi} \tilde{e}_{N}^{(4,1)}+\ldots\right) \frac{O_{0}}{m_{b}^{4}\left(\alpha_{s}\left(\mu_{c}\right) C_{F}\right)^{6}},
$$

where $\mu_{c}$ is the scale in the condensate corrections. We observe that the leading term is proportional to a large negative power of the strong coupling $\alpha_{s}^{-4}\left(\mu_{c}\right)$ and its size therefore grows rapidly with increasing $\mu_{c}$. The NLO correction is of the form $\alpha_{s}^{-3}\left(\mu_{c}\right)\left[c_{N}^{(4,1)}-8 \beta_{0} \ln \left(N \mu_{c} /\left(m_{b} \alpha_{s}\left(\mu_{c}\right) C_{F}\right)\right)\right]$, where the logarithm originates from the potential corrections computed in [22] and has the right prefactor needed to cancel the scale dependence at NLO, and $c_{N}^{(4,1)}$ is independent of $\mu_{c}$ and only partially known due to the missing ultrasoft corrections that appear at NLO. We note that the constant term at NLO is less scale dependent $\left(\propto \alpha_{s}^{-3}\left(\mu_{c}\right)\right)$ than the LO term $\left(\propto \alpha_{s}^{-4}\left(\mu_{c}\right)\right)$, contrary to the familiar situation where the leading order is proportional to a positive power of the strong coupling. This allows us to determine the preferred choice for the scale $\mu_{c}$ from the convergence of the perturbative expansion from the potential part of the NLO correction alone.

\subsection{The $\Upsilon(1 S)$ and $\Upsilon(2 S)$ masses}

The perturbative contribution to the $\Upsilon(1 S)$ mass can easily be evaluated with the public library QQbar_Threshold [17,26] and takes the form

$$
M_{\Upsilon(1 S)}^{\text {pert }}(\mu=3 \mathrm{GeV})=(9366+82+4-27) \mathrm{MeV} .
$$

in the PS mass scheme [27] with the input $m_{b}^{\mathrm{PS}}=4.532_{-0.039}^{+0.013} \mathrm{GeV}$ from $[8,28]$. The scale dependence is shown in the left plot of Figure 5. The right plot shows the scale behaviour of the condensate contributions with the bands being spanned by the range $\tilde{e}_{1}^{\left(4,1_{\text {us }}\right)} \in[-25,25]$ for the missing 

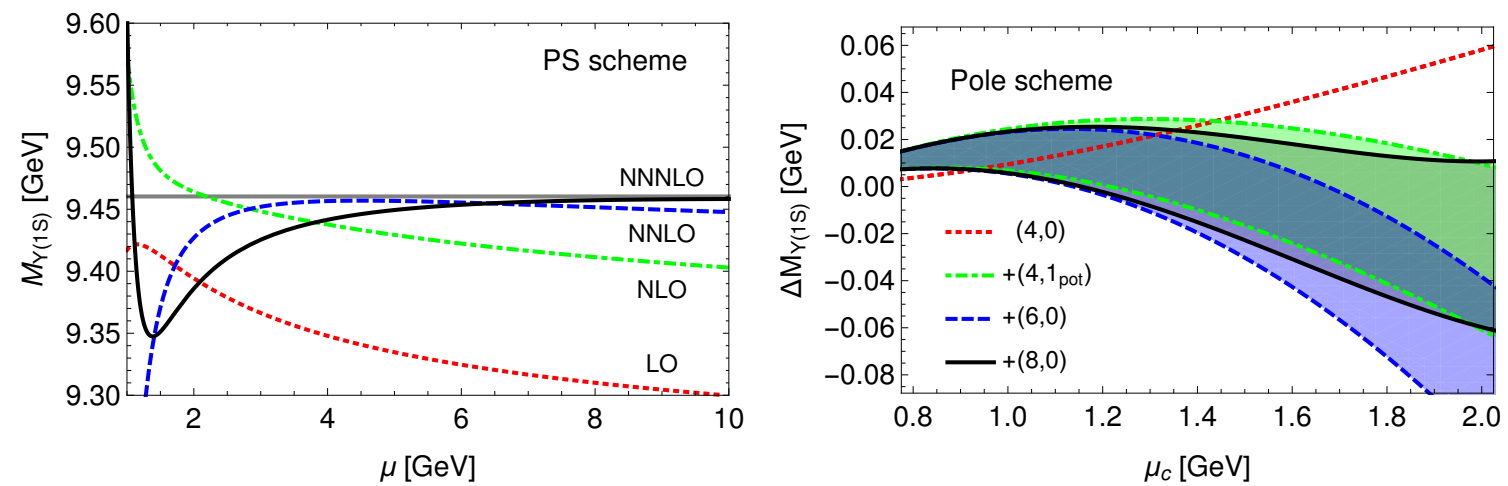

Figure 5: The left plot shows the perturbative contribution to the mass of the $\Upsilon(1 S)$ resonance. The curves in the right plot show the effects of adding the condensate contributions $(i, j)$ cumulatively, where $i$ and $j$ denote the dimension and perturbative order. The bands are spanned by the uncertainty from the missing NLO ultrasoft correction at dimension four which is estimated with $-25 \leq \tilde{e}_{1}^{\left(4,1_{\text {us }}\right)} \leq 25$.

ultrasoft contribution at NLO. The crucial observation is that the convergence of the dimension four contribution gives a clear preference for scales of the order of $1.2 \mathrm{GeV}$ - independently of the exact value of the missing ultrasoft term. Taking this as the central scale choice we also observe good convergence of the higher-dimensional contributions

$$
\Delta M_{\Upsilon(1 S)}^{\text {cond }}\left(\mu_{c}=1.2 \mathrm{GeV}\right)=\left[(17-3) \frac{O_{0}}{O_{0}^{\text {SVZ }}}-4 \frac{O_{1}}{O_{1}^{\text {naive }}}+1 \frac{O_{2}}{O_{2}^{\text {naive }}}\right] \mathrm{MeV} .
$$

The sum of the perturbative and condensate contributions

$$
\begin{aligned}
M_{\Upsilon(1 S)} & =9437_{-114}^{+61} \mathrm{MeV} \\
& =9437_{-74}^{+28}(\mu)_{-75}^{+25}\left(m_{b}\right)_{-1}^{+0}\left(\alpha_{s}\right) \pm 9\left(m_{c}\right) \pm 36\left(\mu_{c}, \text { us }\right)_{-14}^{+29}\left(O_{0}\right)_{-18}^{+4}\left(O_{1}\right)_{-1}^{+10}\left(O_{2}\right) \mathrm{MeV},
\end{aligned}
$$

agrees well with the experimental value $M_{\Upsilon(1 S)}^{\exp }=9460.30 \pm 0.26 \mathrm{MeV}$. Thus finding that all uncertainties are under control, one can extract the value of the bottom-quark mass from $M_{\Upsilon(1 S)}$. The result

$$
\left.\bar{m}_{b}\left(\bar{m}_{b}\right)=4214 \pm 37 \text { (pert. }\right)_{-22}^{+20} \text { (non-pert.) } \mathrm{MeV}=4214_{-43}^{+42} \mathrm{MeV}
$$

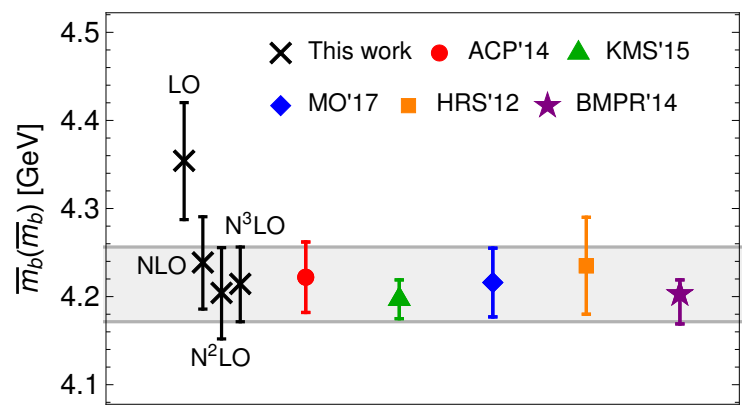

Figure 6: Our value (3.5) for the bottom-quark $\overline{\mathrm{MS}}$ mass compared to other recent results from the spectrum (ACP'14 [29,30], KMS'15 [31], MO'17 [32]) and sum rules (HRS'12 [33], BMPR'14 [8, 28]). 

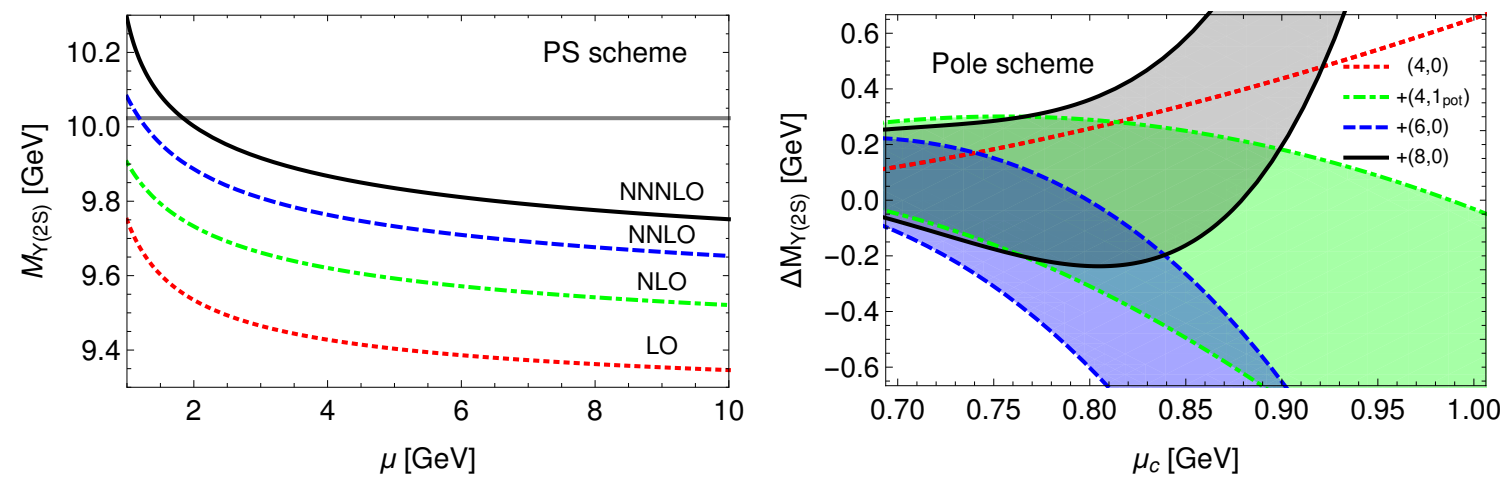

Figure 7: The same as Figure 5 but for the $\Upsilon(2 S)$ mass.

is in good agreement with other recent determinations as shown in Figure 6. While the bulk of the uncertainty is of perturbative origin, the non-perturbative part is non-negligible and pushes the total uncertainty up to $1 \%$.

Repeating the analysis for the $\Upsilon(2 S)$ mass, we first note the the convergence of the perturbative contribution is a lot worse

$$
M_{\Upsilon(2 S)}^{\mathrm{pert}}(\mu=2 \mathrm{GeV})=(9534+198+154+116) \mathrm{MeV} .
$$

Figure 7 demonstrates that this observation is independent of the choice of scale. The condensate contribution is shown in the right plot and shows no sign of convergence, even for very low scales

$$
\Delta M_{\Upsilon(2 S)}^{\text {cond }}\left(\mu_{c}=0.8 \mathrm{GeV}\right)=\left[(258-267) \frac{O_{0}}{O_{0}^{\text {SVZ }}}-293 \frac{O_{1}}{O_{1}^{\text {naive }}}+365 \frac{O_{2}}{O_{2}^{\text {naive }}}\right] \mathrm{MeV} .
$$

Thus, it is currently not possible to reliably estimate the non-perturbative contribution for the excited bottomonium states. Assuming instead $\Lambda_{\mathrm{QCD}} \sim m_{b} v^{2} \ll m_{b} v$ the corrections take the form of non-local condensates, but no phenomenological study exists for this approach.

\subsection{The non-relativistic moments}

The non-relativistic moments $\mathscr{M}_{n}$ with $n \gtrsim 8$ are defined as

$$
\mathscr{M}_{n} \equiv \int_{0}^{\infty} d s \frac{R_{b}(s)}{s^{n+1}}=\left.\frac{12 \pi^{2}}{n !}\left(\frac{d}{d q^{2}}\right)^{n} \Pi_{b}\left(q^{2}\right)\right|_{q^{2}=0},
$$

where $\Pi_{b}$ is the bottom-quark contribution to the vacuum polarization and $R_{b}(s)=12 \pi \operatorname{Im} \Pi_{b}(s+$ $i \varepsilon)$ is the normalized $b \bar{b}$ production cross section in $e^{+} e^{-}$collisions. For the moments $1 / \sqrt{n}$ determines the typical size of the bottom-quark velocity $v$ and the condensate expansion is in powers of $n \Lambda_{\mathrm{QCD}} / m_{b}$. The perturbative NNNLO result and the leading dimension-four contribution for the moments have been obtained and discussed in $[8,28]$. Here, we only focus on the condensate corrections which are shown in Figure 8. The scale dependence of the leading-order dimensionfour contribution is given in the upper left plot for a number of moments with $n$ between 8 and 24 . Including the partial NLO corrections from single insertions of the NLO Coulomb potential yields 

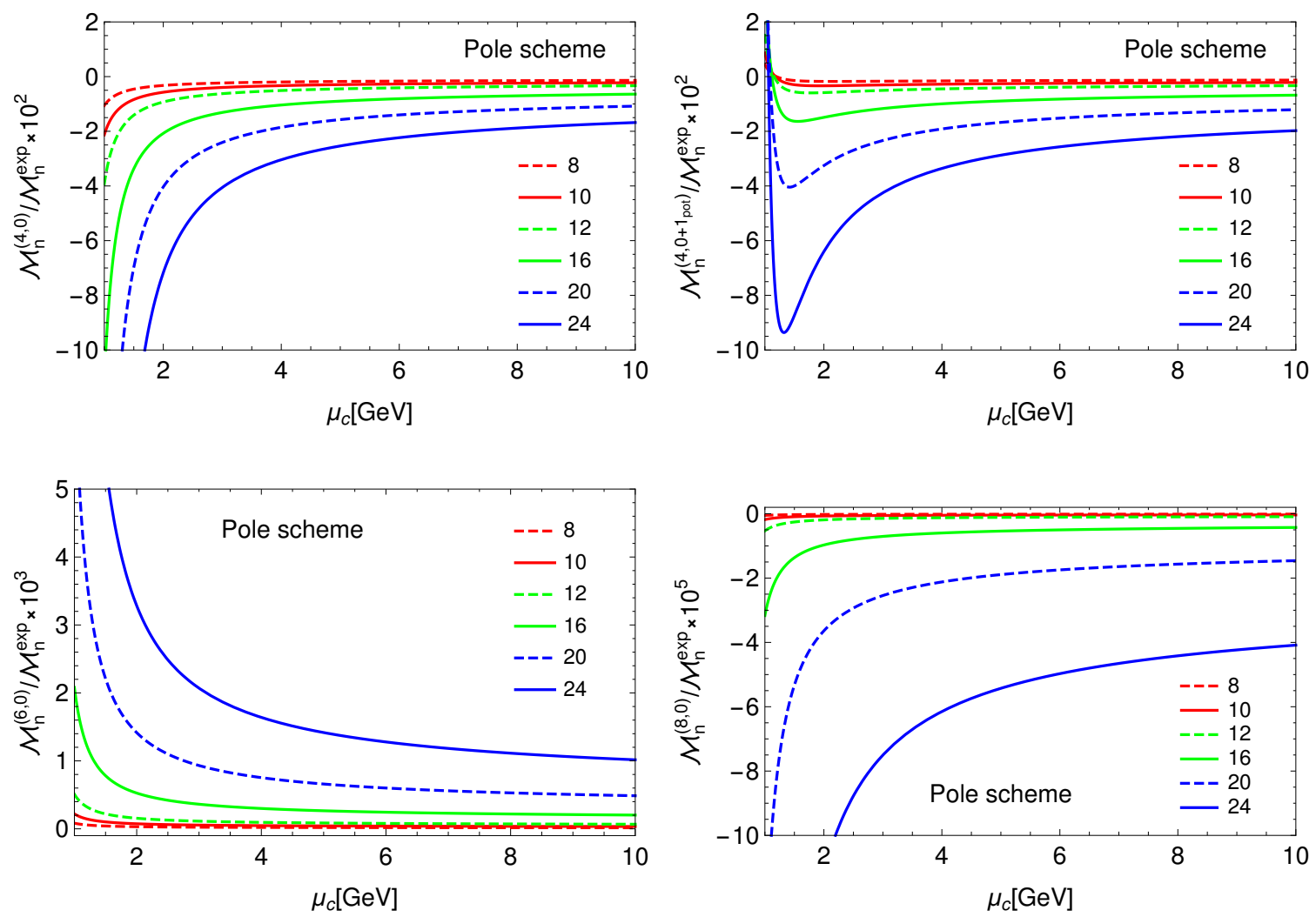

Figure 8: Relative condensate corrections to various non-relativistic moments. The upper panel shows the dimension-four contribution at LO and partial NLO rescaled by a factor of 100. The dimension-six and eight corrections, rescaled by factors of $10^{3}$ and $10^{5}$, respectively, are shown below.

the plot on the upper right. We observe good convergence and a significant reduction of the scale dependence with stable behaviour in the entire range between 1 and $10 \mathrm{GeV}$. From naive power counting we expect the condensate expansion to break down for $n \approx 16$ where $n \Lambda_{\mathrm{QCD}} \approx m_{b}$ and the relative dimension-four contribution is of the order $1 / n \approx 0.06$ due to the $v \sim 1 / \sqrt{n}$ suppression for each of the dipole terms in (2.4). Choosing a low scale $\mu_{c}=1.5 \mathrm{GeV}$ the dimension-four contribution indeed suggests a breakdown for $n$ around 20. The dimension-six and eight corrections shown in the lower panel of Figure 8 are however tiny compared to the dimension-four terms for all considered values of $n$ (note the different rescaling factors in the plots). We conjecture that the reason is that the moments are in fact an off-shell quantity defined at $q^{2}=0$ far away from the physical cut at $q^{2} \geq M_{\Upsilon(1 S)}^{2}$, and the off-shellness provides an infrared cutoff on the higher-dimensional contributions. Therefore, it naively appears that moments up to very high $n$ can be reliably described within the local condensate expansion.

However, the sum rule relies on quark-hadron duality which must be questioned when the moments are completely saturated by the lowest-lying state as is the case for $n=20$ with $95 \%$ of the experimental moment being due to $\Upsilon(1 S)$ [8]. Violations of quark-hadron duality can manifest as terms of the form $\exp \left(-m_{b} /\left(n \Lambda_{\mathrm{QCD}}\right)\right)$ which have a trivial Taylor expansion and therefore do not show up in the local condensate expansion. Such terms can be caused by the emission of 
many soft gluons ${ }^{1}$ which together carry a hard momentum fraction and can therefore reduce the off-shellness of the $b \bar{b}$ system. Thus, we expect that the off-shellness of the moments does not suppress violations of quark-hadron duality. Given that the dimension-four term suggests that the expansion breaks down for $n \approx 20$, we expect that duality violations become relevant in this regime where $\exp \left(-m_{b} /\left(n \Lambda_{\mathrm{QCD}}\right)\right)$ becomes order unity. Therefore, such large values of $n$ should not be used for the determination of the bottom-quark mass. For $n \approx 10$ duality violations are however exponentially suppressed and the condensate corrections are tiny which clearly demonstrated the reliability of a purely perturbative approach.

\section{Conclusions}

I have presented results at higher orders in the local condensate expansion for bottomonium. For the $\Upsilon(1 S)$ mass good convergence is obtained for the scale choice suggested by the convergence of the potential NLO corrections. Therefore it is possible to extract the bottom-quark mass from the experimental value for $M_{\Upsilon}(1 S)$ which yields the result (3.5). While the qualitative conclusion about the stability of the condensate expansion seems rather robust, the size of the condensate contribution is affected by sizable uncertainties due to the poorly known condensates, variation of the scale and the missing ultrasoft correction. Therefore, the non-perturbative uncertainty in the determination of the bottom-quark mass is non-negligible.

On the other hand we have shown that non-perturbative effects for the non-relativistic moments $\mathscr{M}_{n}$ of the bottom-pair production cross section are negligible for $n \approx 10$ which clearly makes this the theoretically cleaner approach for the determination of the bottom-quark mass. However, moments with significantly larger values of $n$ are expected to suffer from the quantitatively poorly understood violations of quark-hadron duality.

\section{References}

[1] M. Beneke and V. A. Smirnov, it Asymptotic expansion of Feynman integrals near threshold, Nucl. Phys. B 522 (1998) 321 [hep-ph/9711391].

[2] A. Pineda and J. Soto, Effective field theory for ultrasoft momenta in NRQCD and NRQED, Nucl. Phys. Proc. Suppl. 64 (1998) 428 [hep-ph/9707481].

[3] M. Beneke, A. Signer and V. A. Smirnov, Top quark production near threshold and the top quark mass, Phys. Lett. B 454 (1999) 137 [hep-ph/9903260].

[4] N. Brambilla, A. Pineda, J. Soto and A. Vairo, Potential NRQCD: An Effective theory for heavy quarkonium, Nucl. Phys. B 566 (2000) 275 [hep-ph/9907240].

[5] M. Beneke, Y. Kiyo and K. Schuller, Third-order correction to top-quark pair production near threshold I. Effective theory set-up and matching coefficients, arXiv:1312.4791 [hep-ph].

[6] Y. Kiyo and Y. Sumino, Perturbative heavy quarkonium spectrum at next-to-next-to-next-to-leading order, Phys. Lett. B 730 (2014) 76 [arXiv:1309.6571 [hep-ph]].

[7] M. Beneke et al., Leptonic decay of the $\Upsilon(1 S)$ meson at third order in QCD, Phys. Rev. Lett. 112 (2014) no.15, 151801 [arXiv:1401.3005 [hep-ph]].

\footnotetext{
${ }^{1}$ See [34] for a discussion of these coherent soft fluctuations and duality violations.
} 
[8] M. Beneke, A. Maier, J. Piclum and T. Rauh, The bottom-quark mass from non-relativistic sum rules at NNNLO, Nucl. Phys. B 891 (2015) 42 [arXiv:1411.3132 [hep-ph]].

[9] P. Marquard, J. H. Piclum, D. Seidel and M. Steinhauser, Three-loop matching of the vector current, Phys. Rev. D 89 (2014) no.3, 034027 [arXiv:1401.3004 [hep-ph]].

[10] R. N. Lee, A. V. Smirnov, V. A. Smirnov and M. Steinhauser, Analytic three-loop static potential, Phys. Rev. D 94 (2016) no.5, 054029 [arXiv:1608.02603 [hep-ph]].

[11] M. Beneke, Y. Kiyo and K. Schuller, Third-order correction to top-quark pair production near threshold II. Potential contributions, in preparation.

[12] M. Beneke and Y. Kiyo, Ultrasoft contribution to heavy-quark pair production near threshold, Phys. Lett. B 668 (2008) 143 [arXiv:0804.4004 [hep-ph]].

[13] A. H. Hoang and M. Stahlhofen, The Top-Antitop Threshold at the ILC: NNLL QCD Uncertainties, JHEP 1405 (2014) 121 [arXiv:1309.6323 [hep-ph]].

[14] M. Beneke, J. Piclum and T. Rauh, P-wave contribution to third-order top-quark pair production near threshold, Nucl. Phys. B 880 (2014) 414 [arXiv:1312.4792 [hep-ph]].

[15] M. Beneke et al., Next-to-Next-to-Next-to-Leading Order QCD Prediction for the Top Antitop S-Wave Pair Production Cross Section Near Threshold in $e^{+} e^{-}$Annihilation, Phys. Rev. Lett. 115 (2015) no.19, 192001 [arXiv:1506.06864 [hep-ph]].

[16] M. Beneke, A. Maier, J. Piclum and T. Rauh, Higgs effects in top anti-top production near threshold in $e^{+} e$ annihilation, Nucl. Phys. B 899 (2015) 180 [arXiv:1506.06865 [hep-ph]].

[17] M. Beneke, A. Maier, T. Rauh and P. Ruiz-Femenía, Non-resonant and electroweak NNLO correction to the $e^{+} e^{-}$top anti-top threshold, JHEP 1802 (2018) 125 [arXiv:1711.10429 [hep-ph]].

[18] M. B. Voloshin, On Dynamics of Heavy Quarks in Nonperturbative QCD Vacuum, Nucl. Phys. B 154 (1979) 365.

[19] M. B. Voloshin, Precoulombic Asymptotics for Energy Levels of Heavy Quarkonium, Sov. J. Nucl. Phys. 36 (1982) 143 [Yad. Fiz. 36 (1982) 247].

[20] H. Leutwyler, How to Use Heavy Quarks to Probe the QCD Vacuum, Phys. Lett. $98 B$ (1981) 447.

[21] M. B. Voloshin, Precision determination of $\alpha_{s}$ and $m_{b}$ from $Q C D$ sum rules for $b \bar{b}$, Int. J. Mod. Phys. A 10 (1995) 2865 [hep-ph/9502224].

[22] T. Rauh, Higher-order condensate corrections to $\Upsilon$ masses, leptonic decay rates and sum rules, JHEP 1805 (2018) 201 [arXiv:1803.05477 [hep-ph]].

[23] A. Pineda, Next-to-leading nonperturbative calculation in heavy quarkonium, Nucl. Phys. B 494 (1997) 213 [hep-ph/9611388].

[24] G. S. Bali, C. Bauer and A. Pineda, Model-independent determination of the gluon condensate in four-dimensional SU(3) gauge theory, Phys. Rev. Lett. 113 (2014) 092001 [arXiv:1403.6477 [hep-ph]].

[25] M. A. Shifman, A. I. Vainshtein and V. I. Zakharov, QCD and Resonance Physics: Applications, Nucl. Phys. B 147 (1979) 448.

[26] M. Beneke, Y. Kiyo, A. Maier and J. Piclum, Near-threshold production of heavy quarks with QQbar_threshold, Comput. Phys. Commun. 209 (2016) 96 [arXiv:1605.03010 [hep-ph]]. 
[27] M. Beneke, A Quark mass definition adequate for threshold problems, Phys. Lett. B 434 (1998) 115 [hep-ph/9804241].

[28] M. Beneke, A. Maier, J. Piclum and T. Rauh, NNNLO determination of the bottom-quark mass from non-relativistic sum rules, PoS RADCOR 2015 (2016) 035 [arXiv:1601.02949 [hep-ph]].

[29] C. Ayala, G. Cvetič and A. Pineda, The bottom quark mass from the $\mathbf{\Upsilon}(1 S)$ system at NNNLO, JHEP 1409 (2014) 045 [arXiv:1407.2128 [hep-ph]].

[30] C. Ayala, G. Cvetič and A. Pineda, Mass of the bottom quark from Upsilon(1S) at NNNLO: an update, J. Phys. Conf. Ser. 762 (2016) no.1, 012063 [arXiv:1606.01741 [hep-ph]].

[31] Y. Kiyo, G. Mishima and Y. Sumino, Determination of $m_{c}$ and $m_{b}$ from quarkonium $1 S$ energy levels in perturbative QCD, Phys. Lett. B 752 (2016) 122 Erratum: [Phys. Lett. B 772 (2017) 878] [arXiv:1510.07072 [hep-ph]].

[32] V. Mateu and P. G. Ortega, Bottom and Charm Mass determinations from global fits to $Q \bar{Q}$ bound states at $N^{3} L O$, arXiv:1711.05755 [hep-ph].

[33] A. Hoang, P. Ruiz-Femenía and M. Stahlhofen, Renormalization Group Improved Bottom Mass from Upsilon Sum Rules at NNLL Order, JHEP 1210 (2012) 188 [arXiv:1209.0450 [hep-ph]].

[34] M. A. Shifman, Quark hadron duality, hep-ph/0009131. 